\title{
Reply from Honglei Wang, Tai-Shung Chung, Yen Wah Tong, Wenyuan Xie and Fang He
}

\begin{abstract}
Kocherginsky presented a critical analysis of our article (published in Small 8, 2012, pp. 1185-1190) in his paper "Aquaporin, forward osmosis and biomimetic membranes" (in Gen. Physiol. Biophys. 4, 2013, pp. 589-594). However, after a detailed analysis of his arguments, it is easy to recognize that Kocherginsky did not understand the permeability of aquaporin-based biomimetic membranes nor the membrane structures in our article. It is clear that Kocherginsky's interpretation of our article was based on wrong calculations and assumptions that are not justified. Herein, the main points mentioned in Kocherginsky's paper are summarized and a point-by-point response detailed showing how the errors have arisen.
\end{abstract}

\section{Membrane selectivity}

Kocherginsky claimed that the calculation of water selectivity is wrong (p. 590, left column, line 41-53). Unfortunately, it seems that he either misunderstood the definition of selectivity or the calculation done in our paper. Kocherginsky claimed that when membrane separate pure water and $2 \mathrm{M} \mathrm{NaCl}$, the selectivity calculated based on water flux and reverse salt flux is more than 46,000. The membrane selectivity is used to compare the separation capacity which is always measured when two molecules move in the same direction. The selectivity calculation based on a water flux of $142 \mathrm{l} / \mathrm{m}^{2} / \mathrm{h}$ and a salt reverse flux of $10 \mathrm{~g} / \mathrm{m}^{2} / \mathrm{h}$ will overestimate the selectivity, because water and salt move in opposite directions in this case. In other words, the transport of salt and water compete with each other not only based on membrane selectivity, but also due to movement direction. Therefore, the selectivity in Table 1 is calculated based on the experiment in which sucrose solution was used as the draw solute with a feed of a salt solution. In this case, water and salt will compete with each other to move to the sucrose solution side, and thus the selectivity calculated in this experiment is closer to the literature data as reported by those stated in Kocherginsky's paper.

\section{Arrhenius equation}

Kocherginsky is questioning the Arrhenius equation (p. 591, right column, second paragraph from the bottom). The question is not targeted towards the well-known application of Arrhenius equation to estimate the activation energy, but instead it is about how to estimate the activation energy of a biomimetic membrane and how to correlate the activation energy of a pore-spanning membrane with vesicles.

If Kocherginsky is familiar with the leading publications by Borgnia et al. (1999) and Kumar et al. (2007), the activa- tion energy of vesicular membranes has been estimated using the correlation of vesicle permeability $P_{f}$ and T

$$
P_{f}=\frac{k}{\left(S / V_{0}\right) V_{w} \Delta_{o s m}}
$$

where $P_{f}$ is the osmotic water permeability $(\mathrm{m} / \mathrm{s}), S$ is the vesicle surface area $\left(\mathrm{m}^{2}\right), V_{0}$ is the initial vesicle volume $\left(\mathrm{m}^{3}\right), V_{w}$ is the partial molar volume of water $(0.018 \mathrm{l} / \mathrm{mol}), \Delta_{\text {osm }}$ is the osmolarity difference that drives the shrinkage of the vesicles (osmol/l), and $k$ is the initial rate constant $\left(\mathrm{s}^{-1}\right)$. The activation energy of the biomimetic bilayer membrane was calculated by correlation of $k$ and $\mathrm{T}$ in Borgnia's paper (Figure 7) (Borgnia et al. 1999) and Kumar's paper (Figure 3) (Kumar et al. 2007).

In our paper, the intention of Eq. 5 is to prove that $J_{w}$ (water flux of the pore-spanning membrane) is related to $P_{f}$, and therefore, by correlating $J_{w}$ and $\mathrm{T}$, the activation energy of pore-spanning membrane can be estimated.

\section{Flux of substrate}

Kocherginsky also questioned the flux of the substrate membrane (p. 590, right column, line 1-25). This should be noted in relation to Eq. 1 on page 590 that is used estimate a ballpark figure of the osmotic permeability of the porous support. While it is true that Eq. 1 holds for the diffusiondominated transport, but in this case, it is evident that in the larger pore sizes of the porous support membrane used in our work, where the pore size is at least sub-micron range, the transport mechanism is no longer limited by diffusion. It is common knowledge that fluid transport through membrane will be controlled by two mechanisms, namely, convection and diffusion (Byron Bird et al. 2002). In microfiltration (MF) and ultrafiltration (UF) membranes, diffusion process through large pore membranes is typically negligible relative to convection. As the substrate membrane in our paper is 
a UF membrane with pore size of at least $50 \mathrm{~nm}$, convection dominates the membrane flux. However, Kocherginsky's theoretical estimation on the water flux of the substrate (p. 590, Eq. 1 and Eq. 2) is based on a diffusion mechanism which is only feasible in non-porous membranes such as reverse osmosis (RO) membranes. Therefore, Kocherginsky's calculations greatly underestimate the water flux of the substrate membrane. In addition, we have measured the pure water permeability (PWP) of the substrate membrane and have found it to be above $500 \mathrm{LMH} /$ bar.

\section{Calculation of the theoretical water flux}

Kocherginsky is questioning the theoretical water flux (p. 590 (right column, line 27-53) - p. 591 (left column, line 1-7)). It should be noted that the stopped-flow experiment only measures vesicle permeability, but it could not tell whether aquaporins are evenly distributed on the vesicle surface. Kocherginsky's estimation is technically correct if the aquaporin is evenly distributed. In reality, the aquaporin proteins could form a high density region and a low density region. When we calculated the theoretical membrane flux, we tend to push the case to an extreme case. In such cases, all the proteins could locate in the pore-spanning membrane area. This had been also our dream when such membrane was developed.

Kocherginsky claimed that the water permeates through the membrane channels into the unstirred layers limited by the vesicle size $(150 \mathrm{~nm})$ (p. 590 (right column, line 52-53) - p. 591 (left column, line 1)). Kocherginsky did not even understand our membrane structure. In our article, we use a pore-spanning biomimetic membrane, where the vesicles are ruptured and are deposited as biomimetic bilayers on the support membrane. Therefore, there is no such "unstirred layer limited by the vesicle size" as mentioned in Kocherginsky's paper.

\section{Aquaporin permeability}

We believe that Kocherginsky did not take into account that the permeability of aquaporin-incorporated vesicles depends on the incorporation ratio of aquaporins to block copolymers. As there is a much lower incorporation ratio in oocyte cells as compared to biomimetic vesicles, the calculations would indicate a lower flux as he has shown. However, the permeability of each aquaporin monomer or tetramer unit is all in the similar range of $10 \times 10^{-14} \mathrm{~cm}^{3} / \mathrm{s}$, whether they are in the oocytes or in the vesicles.

\section{Membrane selectivity assumptions}

It is arguable that Eq. 8 in Kocherginsky's paper does not show dependence in the concentration of the draw solution in the forward osmosis set-up. This is clearly counter-intuitive and against experimental results found in our work and others. We urge the author to demonstrate the derivation of Eq. 8 and the various assumptions involved in the derivation, which might limit the applicability of Eq. 8 for forward osmosis.

In addition, the value of $\mathrm{D}_{w} /\left(\mathrm{K}\left({ }_{w, 1}\right) \mathrm{D}\left({ }_{w, 1}\right)\right)$ seems to be too convenient to be assumed to be $1 / 30$. The author should provide a source to substantiate his assumption for this reference value. As numerous well-established literatures has pointed out that the osmotic transport inside aquaporin- $Z$ porous channel is approaching the diffusion limit, it can be adequately assumed that $\mathrm{D}_{w} \approx \mathrm{D}\left({ }_{w, l}\right)$ (David et al. 2010), and to follow the author's assumption on $\mathrm{K}\left({ }_{w, 1}\right)$ being slightly less than unity, it is arguable that the value of $\mathrm{D}_{w} /\left(\mathrm{K}\left({ }_{w, 1}\right) \mathrm{D}\left({ }_{w, 1}\right)\right)$ should be 1 instead of $1 / 30$.

Furthermore, the Eq. 4 in Kocherginsky's paper is based on the wrong assumption that the water permeability of the whole membrane is limited by the support layer. It is shown above that Kocherginsky greatly underestimated the permeability of the substrate membrane used and thus we show that the water permeability of the support membrane is high. Therefore, this should not be the limitation of the whole membrane permeability. As a result, in Kocherginsky's paper, Eq. 6, 7 and 8, which are all based on Eq. 4, would thus be meaningless or provide highly underestimated values.

Honglei Wang, Tai-Shung Chung, Yen Wah Tong, Wenyuan Xie and Fang He

Department of Chemical and Biomolecular Engineering, National University of Singapore, Singapore 119260, Singapore E-mail: chetyw@nus.edu.sg

\section{References}

Borgnia M. J., Kozono D., Calamita G., Maloney P. C., Agre P. (1999): Functional reconstitution and characterization of AqpZ, the E. coli water channel protein. J. Mol. Biol. 291, 1169-1179 http://dx.doi.org/10.1006/jmbi.1999.3032

Byron Bird R., Stewart W. E., Lightfoot E. N. (2002): Transport Phenomena. 2nd ed., John Wiley \& Sons, Inc., New York Kocherginsky N. (2013): Aquaporin, forward osmosis and biomimetic membranes. Gen. Physiol. Biophys. 4, 589-594

Kumar M., Grelakowski M., Zilles J., Clark M., Meier W. (2007): Highly permeable polymeric membranes based on the incorporation of the functional water channel protein Aquaporin Z. Proc. Natl. Acad. Sci. USA 104, 20719-20724 http://dx.doi.org/10.1073/pnas.0708762104

Savage D. F., O'Connell J. D., Miercke L. J. W., Finer-Moore J., Stroud R. M. (2010): Structural context shapes the aquaporin selectivity filter. Proc. Natl. Acad. Sci. USA 107, 17164-17169 http://dx.doi.org/10.1073/pnas.1009864107

Wang H., Chung T.-S., Tong Y. W. (2013b): Study on water transport through a mechanically robust Aquaporin $\mathrm{Z}$ biomimetic membrane. J. Memb. Sci. 445, 47-52 http://dx.doi.org/10.1016/j.memsci.2013.05.057 